\title{
Single-Staged Transoral Intra-Lesional Excision of Aneurysmal Bone Cyst of Axis (C2) Vertebra and Temporary Stabilization of Upper Cervical Spine in an Adolescent: A Case Report
}

\author{
Khan $E S^{a, b}$, Hazwan AWc, Sharifudin $M A^{b}$, Ramos $J^{a, d}$, Pingel $A^{a}$, Kandziora $F^{a}$ \\ a'Zentrum für Wirbelsäulenchirurgie und Neurotraumatologie, Berufsgenossenschaftliche Unfallklinik, \\ Friedberger Landstrasse 430, 60389 Frankfurt am Main, Germany. \\ bepartment of Orthopaedics, Traumatology and Rehabilitation, Kulliyyah (Faculty) of Medicine, \\ International Islamic University, Jalan Sultan Ahmad Shah, 25200 Kuantan, Pahang, Malaysia. \\ 'Department of Orthopaedic \& Traumatology, Hospital Tengku Ampuan Afzan, Pahang, Malaysia. \\ ${ }^{d}$ Centro Medico Puerta de Hierro, Boulevard Puerta de Hierro 5150, Torre C PB., C.P. 45116, Zapopan, \\ Jalisco, Mexico.
}

\section{ABSTRACT}

Aneurysmal bone cyst $(A B C)$ infrequently occurs within the upper cervical vertebrae. Various therapeutic options have been reported in the literature. We would like to share our experience in managing a case of a 16-year-old girl diagnosed with $A B C$ at the body of axis (C2) vertebra. Serious attention had to be given on the stability of the cervical spine following tumour resection, which can be affected by the mode of treatment chosen. Instability can have a detrimental effect on the cervical spine, in which case may necessitate further surgery. We performed a single-staged intra-lesional curettage via a transoral approach and temporary non-fusion posterior stabilization of C1 lateral mass screw and C2 pedicle screw. The implants were removed after six months once ossification of $\mathrm{C} 2$ has taken place to regain full motion of the neck. There was no evidence of recurrence or instability of the cervical spine three years following surgery.

KEYWORDS: aneurysmal bone cyst; lytic lesion; cervical spine; posterior instrumentation; transoral approach

\section{INTRODUCTION}

Aneurysmal bone cyst $(A B C)$ is a benign primary bone tumour which predominantly affects patients in the first two decades of life, ${ }^{1-7}$ with slightly higher incidence in females. ${ }^{8,9}$ Its prevalence is 1.4 cases per $100 \quad 000$ individuals constituting approximately $1 \%$ of all bone tumours. ${ }^{5,8}$ Most cases involve the metaphysis of long bones and flat bones. ${ }^{1,4-6,9}$ It constitutes $10 \%$ to $30 \%$ of all primary spine tumours ${ }^{1,4-6}$ with $30-45 \%$ involving the thoracic and lumbar spine, and $2-20 \%$ affecting the cervical spine $^{1-6}$ and rarely affects the upper cervical. ${ }^{3}$

Dr. Hazwan Ab. Wahid

Department of Orthopaedic \& Traumatology, Hospital Tengku Ampuan Afzan Pahang, Jalan Tanah Putih, 25100 Kuantan, Pahang, Malaysia Tel. No. +6012-9782397

Email: hazwan.ab.wahid@gmail.com
We report a case of a 16-year-old girl who was treated for $A B C$ involving the body of axis vertebra (C2) with an intra-lesional curettage through a transoral approach followed by non-fusion temporary posterior stabilization of $\mathrm{C} 1 \mathrm{C} 2$.

\section{CASE REPORT}

A 16-year-old girl presented with sudden onset of upper neck pain following a hyperflexion injury during sporting activity. The pain was localized and partially relieved with analgesics. Following the injury she was able to ambulate independently. There was tenderness over the upper cervical on deep palpation and active neck motion was restricted. Neurological examination were normal. Plain radiographs revealed loss of cervical lordosis An expansile lytic lesion evidence by thinning of cortical wall occupying the right half of the vertebral 
body extending into the odontoid was noted on CT imaging (Figures 1a, 1b). MR imaging revealed a cystic heterogenous hyperintense lesion on T2weighted images and further enhancement post contrast (Figures 1c-f). The classical fluid-fluid levels were not obviously seen on neither CT nor MRI. A CT guided transoral percutaneous biopsy was done and histopathological examination supports the diagnosis of $A B C$ (Figure 2).

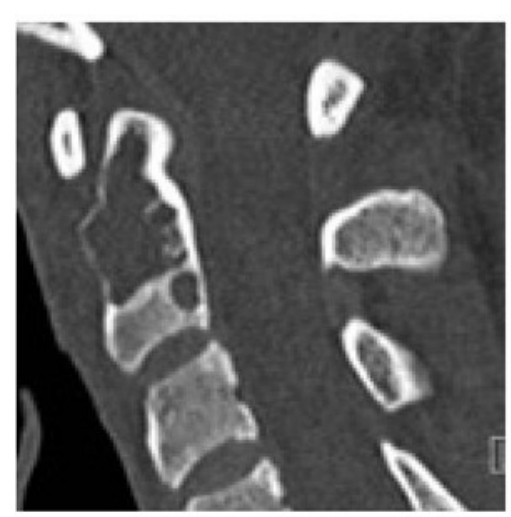

Figure 1a

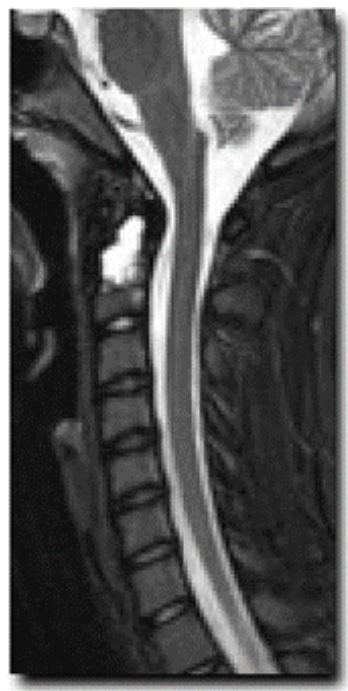

Figure 1c

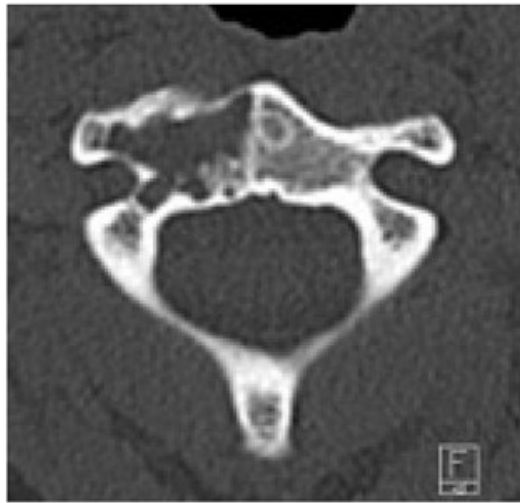

Figure 1b

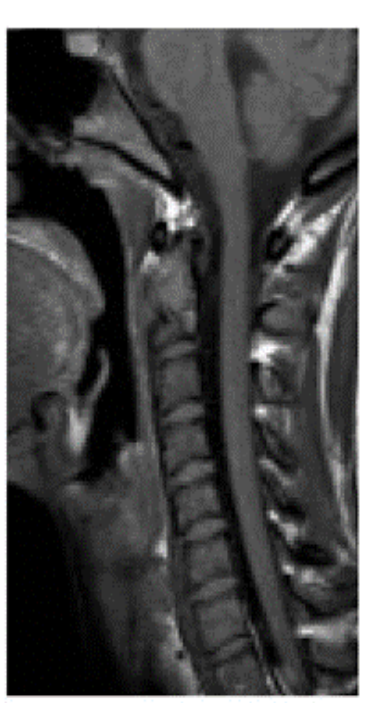

Figure 1d

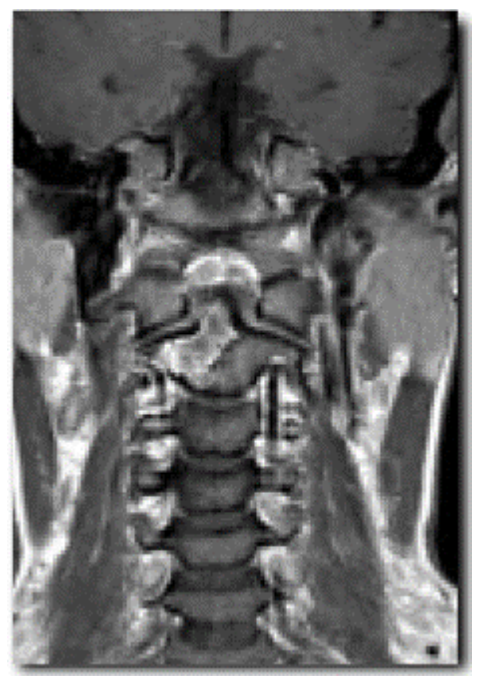

Figure 1e

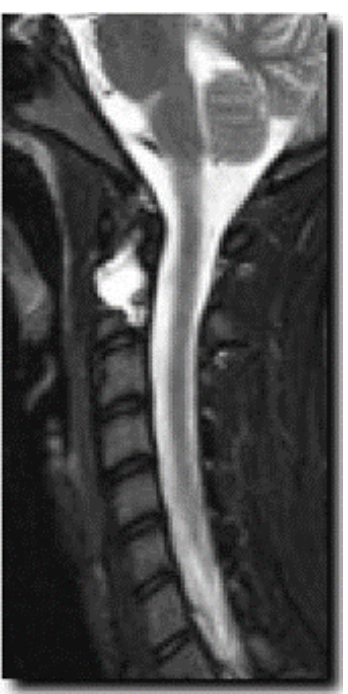

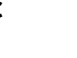

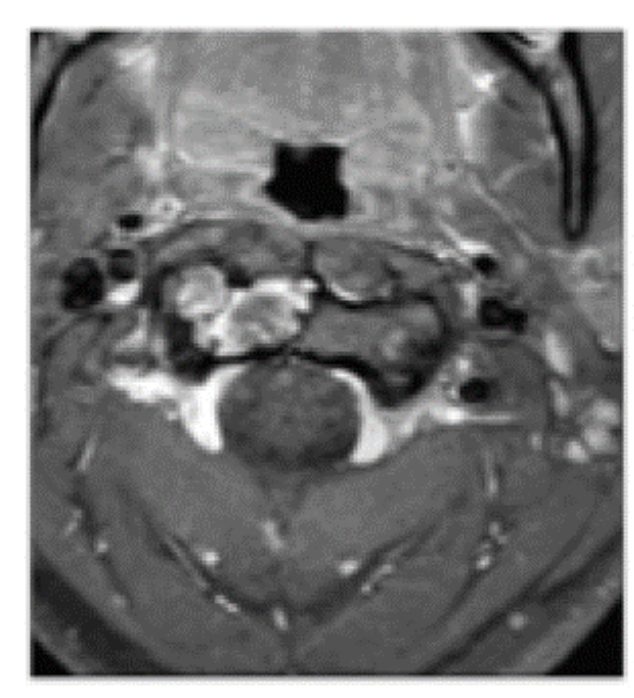

Figure 1f 


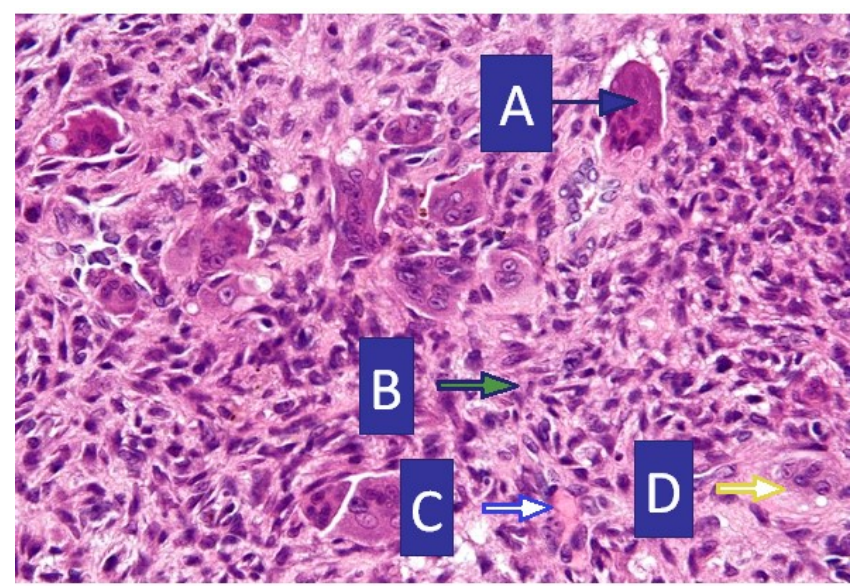

Figure 2: Histopathological examination under H\&E stain $\times 200$ magnification shows a cellular tumour composed of $(A)$ osteoclast-like multinucleate giant cells surrounded by (B) fibroblasts, (C) osteocytes and (D) a few capillary-sized blood vessels highly suggestive of aneurysmal bone cyst.

We performed an intra-lesional gross total excision via transoral approach which gave good exposure, followed by a non-fusion temporary posterior atlantoaxial stabilization of C1C2. A standard transoral surgical technique was used to approach the body of C2. An ultrasonic bone scalpel was used to make a fenestrae via the cortical body to reduce blood loss during the procedure and to obtain a better surgical view. The lesion was removed by curettage, followed by a high-speed burr until healthy bone was grossly visualized. The completion of removal was evaluated using an intra-operative CT scan. The right vertebral artery was decompressed in view of its close proximity with the lesion. The void space created was filled with autologous cancellous bone graft harvested from the iliac crest as it will accelerate ossification. The second procedure was performed in prone position. A non-fusion temporary stabilization technique of $\mathrm{C} 1$ lateral mass and $\mathrm{C} 2$ pedicle screw fixation were used (Figures 3a-d).

Post-operatively, her neck pain was completely resolved after 3 months of surgery. Six months post-surgery, CT images showed homogenous bony appearance suggestive of good bony ossification with no evidence of recurrence (Figures 4a-d). Implants were then removed. Around six weeks post removal of implants, patient regained full range of motion of her neck on clinical assessment during follow-up. She was followed up at our institution up to 14 months following surgery before continuing her routine follow-ups at the local hospital. No recurrence was noted up to three years of follow up.

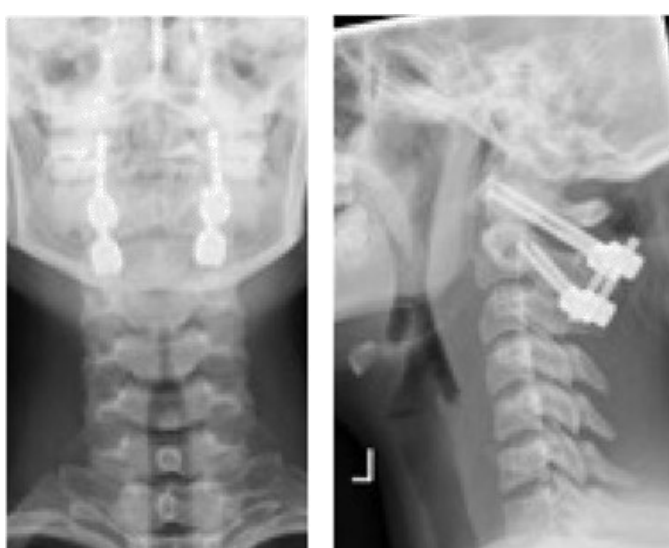

Figure $3 a$ \& $3 b$
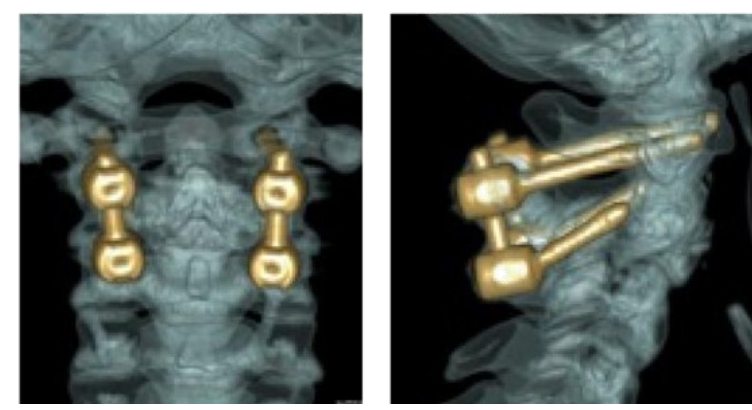

Figure 3c\& $3 d$

Figure 3: Post-operative imaging; $(a, b)$ plain radiograph and $(\mathrm{c}, \mathrm{d}) \mathrm{CT}$ reconstruction images showing posterior instrumentation of $\mathrm{C} 1$ lateral mass screw and $\mathrm{C} 2$ pedicle screw.
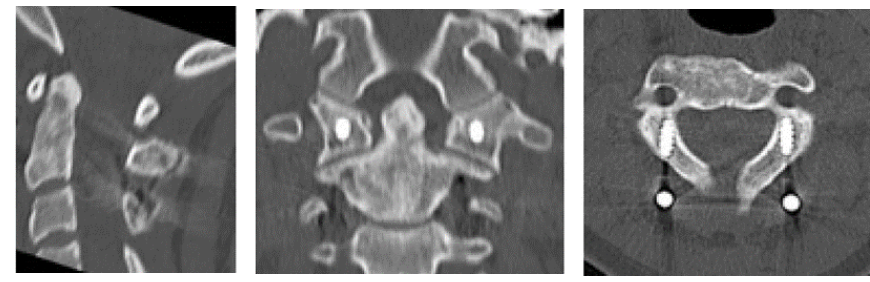

Figure $4 a, 4 b \& 4 c$

Figure 4: CT image six month after surgery prior to implant removal showing cyst area filled with bony ossification (a) sagittal, (b) axial and (c) coronal view with no evidence of recurrence.

\section{DISCUSSION}

$A B C$ is a benign primary bone tumour of unknown aetiology. ${ }^{4-6}$ Although benign, they are locally aggressive. ${ }^{2,4,7-9}$ Some regard it as a pseudotumoural hyperaemic-hemorrhagic lesion secondary to local haemodynamic changes., ${ }^{5,6}$ It may be associated with other primary tumours such as telangiectatic osteosarcoma, haemangioma, giant cell tumour, chondroblastoma, and others; a variant frequently referred to as secondary $A B C .^{2,5,7}$ The natural history of $A B C$ remains hypothetical with various reports on its aggressiveness and progressiveness. ${ }^{4,5,7}$ Spontaneous regression and malignant transformation with and without history of radiation has been reported. ${ }^{1,2,4,8}$ 
Three stages have been described, first as an early lytic phase, followed by a mature phase characterised by an internal septa and peripheral bony shell, and third stage as the calcified stage. ${ }^{10}$ Clinical presentations depend on the localisation of the lesion and its magnitude of spread. ${ }^{5}$ In the cervical spine, the main symptoms are neck pain with restricted neck motion followed by upper limb paraesthesia. Most patients had duration of symptoms of one to two years prior to diagnosis. ${ }^{4,5}$ Our patient was asymptomatic until she had a provocative event that precipitated her neck pain hence it was incidentally diagnosed at an early stage.

CT and MR imaging modalities provides typical features of $A B C$ including expansile lytic lesion with cortical thinning, and presence of fluidfluid level, ${ }^{2,4-7}$ however none are considered pathognomonic as they may also present in other benign and malignant lesions. ${ }^{10}$ Nevertheless, both modalities are important for preoperative planning. ${ }^{2,5,8}$ An atypical feature of imaging as in our patient, advocates histopathological confirmation. ${ }^{7}$

Wide range of treatment options are available, ${ }^{4,8,9}$ from en-bloc or intra-lesional excision with bone grafting, selective arterial embolisation, intra-lesional injection of calcitonin and methylprednisolone, radiotherapy to sclerotherapy. However, optimal treatment remains debatable. Patient's age, presentation, level of vertebral involvement will serve as a guide towards the choice of treatment.

When open surgery is the choice of treatment, the approach depends mainly on the site of pathology. Majority of spinal $A B C s$ involved the posterior elements thus a posterior approach surgery would be more beneficial. ${ }^{2,6,10}$ However, our patient had the lesion exclusively in the body of axis(C2). Insufficient access and higher recurrence rate were reported when resecting anteriorly located lesion using posterior approaches. ${ }^{2,6}$ Hence, we decided to approach the lesion anteriorly via a transoral approach, ${ }^{8}$ which is direct and safe, provided strict pre-operative and intra-operative precautions were followed.

Although an en-bloc resection is optimal for local control and prevention of recurrence, it is not always feasible especially when dealing with a high cervical lesion. ${ }^{4,5,9}$ It is technically demanding and necessitates combination of approaches such as additional transmandibular access following which complex reconstruction is required, leading to a significant restriction of neck motion. ${ }^{9}$ Even then, histopathological results of a series of spinal $A B C s$ following an en-bloc resection revealed presence of tumour residuals at surgical margins. ${ }^{5}$ In another case series, Garg et al. recommended a four-step surgical regime, which include curettage, high-speed burr, electrocautery, and bone grafting. ${ }^{6}$ Complete tumour removals with no local recurrence and spinal deformity in any of the cases were obtained. ${ }^{6}$ Bone curettage alone has been reported to have a fourfold of recurrence rate. ${ }^{7}$ We took a further step in making sure the completeness of our surgery by performing an intra-operative $\mathrm{CT}$ imaging.

It is inevitable that stability would be compromised whenever a complete removal of tumour is the goal of treatment, especially when the lesion is significant in size. Spinal stabilization is then required to provide the stability that has been sacrificed. ${ }^{2,5,7,9}$

In the current case, temporary stabilization of atlantoaxial (C1C2) without fusion was used. This was to allow anterior body ossification to take place since an autograft was used, hence preventing collapse of the body causing instability. The stability of the cervical spine will re-establish once anterior body ossification and consolidation have taken place. Posterior fusion was not necessary since the posterior elements were not involved. Patient will also regain full range of motion once the implants are removed. Important steps in assuring the success of this approach are meticulous soft tissue dissection and not keeping the implants for too long. We removed the implants six months following surgery. CT imaging of the cervical spine prior to removal of implants showed evidence of bony ossification (Figure 4).

As $A B C s$ are highly vascularized lesions, there are recommendations on pre-surgical embolization to minimise blood loss hence a better surgical field. ${ }^{1,2,5-8}$ However, the risks of cord ischemia, brain emboli and dural injury especially in high cervical lesions need to be anticipated. ${ }^{2,6,7}$ Embolization, may be considered as a definitive option for inoperable cases provided there are no concurrent 
pathological fracture, neurological involvement, spinal deformity or instability. Similarly, it may benefit cases of recurrence following repeated surgeries. ${ }^{9}$

Studies on results of intra-lesional injection of calcitonin and methylprednisolone, radiotherapy and sclerotherapy varies and mainly are used in small lesions, inoperable cases, recurrence and lesions involving mainly the long bones, and definitely not a suitable as a standalone treatment in cases of pathological fracture, presence of or impending instability of the spine or presence of cord compression. ${ }^{7}$ Other modalities of treatment besides surgery also have its own significant adverse effects. ${ }^{6,9}$

\section{CONCLUSION}

Management of an upper cervical lesion of $A B C$ remains a surgical challenge. Early diagnosis and intervention before deformity or pathological fractures sets in are the keys to a more successful outcome. Transoral approach for a lesion of the body of C2 vertebra combined with the four-step surgical regime, which includes curettage, highspeed burr, electrocautery and bone grafting is a good technique to achieve an intra-lesional complete resection with minimal risk of recurrence. In preserving full range of motion of the cervical spine, posterior instrumentation without fusion is an excellent option to be considered when posterior elements are not involved.

\section{ACKNOWLEDGEMENTS}

Thanks to the people who were directly and indirectly related with this case report for their relentless support.

Funding: No funding sources

Conflict of interest: None declared

Ethical approval: Not required

\section{REFERENCES}

1. Cottalorda J, Kohler R, Sales de Gauzy J, Chotel F, Mazda K, Lefort G, et al. Epidemiology of aneurysmal bone cyst in children: a multicenter study and literature review. J Pediatr Orthop B 2004; 13:389-94.

2. de Kleuver M, van der Heul RO, Veraart BE. Aneurysmal bone cyst of the spine: 31 cases and the importance of the surgical approach. J

Pediatr Orthop B 1998; 7:286-92.

3. Harrop JS, Schmidt MH, Boriani S, Shaffrey $\mathrm{Cl}$. Aggressive "benign" primary spine neoplasms: osteoblastoma, aneurysmal bone cyst, and giant cell tumor. Spine (Phila Pa 1976) 2009; 34:S3947.

4. Mankin HJ, Hornicek FJ, Ortiz-Cruz E, Villafuerte J, Gebhardt MC. Aneurysmal bone cyst: a review of 150 patients. J Clin Oncol 2005; 23:6756-62.

5. Boriani S, De lure F, Campanacci L, Gasbarrini A, Bandiera $S$, Biagini $R$, et al. Aneurysmal bone cyst of the mobile spine: report on 41 cases. Spine (Phila Pa 1976) 2001; 26:27-35.

6. Garg S, Mehta S, Dormans JP. Modern surgical treatment of primary aneurysmal bone cyst of the spine in children and adolescents. J Pediatr Orthop 2005; 25:387-92.

7. Tsagozis $P$, Brosjo 0 . Current strategies for the treatment of aneurysmal bone cysts. Orthop Rev (Pavia) 2015; 7:6182.

8. Hu Y, Albert TJ, Kepler CK, Ma WH, Yuan ZS, Dong WX. Unstable Jefferson fractures: results of transoral osteosynthesis. Indian J Orthop 2014; 48:145-51.

9. Parmar HN, Agrawal VA, Shah MS, Nanda SN. Locally aggressive aneurysmal bone cyst of $\mathrm{C} 4$ vertebra treated by total en bloc excision and anterior plus posterior cervical instrumentation. J Craniovertebra Junction Spine 2015; 6:130-3.

10. Rajput D, Tungaria A, Jaiswal A, Jain V. Aneurysmal bone cyst of Clivus and C1 C2: case report and review of literature. Turkish Neurosurgery 2012; 22:105-108. 
